\title{
History of phototherapy in neonatal jaundice and the likelihood of childhood asthma
}

\author{
Mohammad Hossein Eslamian ${ }^{\mathbb{*}}$, Mohammad Kazem Sabzehei ${ }^{\circledR}$, Roya Raeisi* ${ }^{\circledR}$, Fatemeh Eghbalian ${ }^{(}$, Abbas Moradi, \\ Mehran Hajiloo
}

Department of Pediatrics, Hamadan University of Medical Sciences, Hamadan, Iran

\section{*Correspondence to \\ Roya Raeisi, Email: r.raisi@ \\ umsha.ac.ir, \\ r_reisi2@yahoo.com}

Received 14 July 2020 Accepted 1 Oct. 2020 Published online 16 Oct. 2020

Keywords: Phototherapy, Neonatal jaundice, Childhood asthma

\begin{abstract}
Introduction: The increased risk for childhood asthma in children suffering from hyperbilirubinemia, and those who underwent phototherapy has attracted enormous interest.

Objectives: In this study, we attempted to explain the relationship between childhood asthma and phototherapy. Patients and Methods: This case-control study was performed on children aged between 2 and 14 years old with a definitive diagnosis of childhood asthma. A sex and age-matched group of children without any pieces of evidence, of asthma or other respiratory problems were also selected as the control group. The hospital's recorded files were the primary sources for the information about demographics, history of asthma in neonates or his/her parents, history and time of phototherapy as well as the data on neonatal hyperbilirubinemia.

Results: In total, 69 children who have asthma and 71 non-asthmatic children as the controls were included in this study. Moreover, these two groups were matched for baseline variables. Comparing the history of phototherapy between the asthmatic and non-asthmatic groups showed a higher rate of phototherapy in the former group $(37.7 \%$ versus $11.3 \% ; P<0.001)$. Accordingly, the risk of asthma could be increased according to the history of phototherapy, up to four times $(\mathrm{OR}=4.76,95 \% \mathrm{Cl}: 1.97-11.51, P<0.001)$.

Conclusion: The risk of childhood asthma can increase if a child had the previous phototherapy. This causal effect is independent of the degree or the age of occurring hyperbilirubinemia, which is the main indication for phototherapy.
\end{abstract}

\section{Introduction}

Citation: Eslamian $\mathrm{MH}$ Sabzehei MK, Raeisi R, Eghbalian F, Moradi A, Hajiloo M. History of phototherapy in neonatal jaundice and the likelihood of childhood asthma. Immunopathol Persa. 2022;8(1):e13. DOI:10.34172/ ipp. 2022.13
Nowadays, Chronic and non-communicable diseases are considered as the leading causes of mortality and disability worldwide that result in a high economic burden on both governments and the communities (1). More than $75 \%$ of all deaths worldwide are due to non-communicable diseases that three-fourth of this burden is reported to be in developing countries (2,3). Childhood asthma is now a major chronic disorder with an upward trend in recent decades $(4,5)$. This increasing trend is originally associated both with the changes in genetic variants and some triggering environmental factors (6). The disease has a damaging effect on the development of the respiratory tract, and these undesirable changes remain with the person until adulthood. Despite significant advances in diagnosis and treatment of childhood asthma, the disabilities and disease-related complications, especially in low- income countries, remains high. Accordingly, this has led to a strong tendency for identifying the risk factors for the prevention of asthma in children. Recently, a significant link has been found between the

\section{Key point}

The risk of childhood asthma can increase if a child had the previous phototherapy

likelihood of childhood asthma and neonatal phototherapy (despite its benefits on the treatment of hyperbilirubinemia) $(7,8)$. It seems that phototherapy for neonatal icterus may stimulate neonate immune system by affecting T-lymphocytes production, which consequently leads to disturbance in neonatal immune system development (9). In this regard, inhibition of $\mathrm{T}$ lymphocyte function may result in a reduction in $\mathrm{T}$ helper 2 to $\mathrm{T}$ helper 1 lymphocyte transformation that may lead to childhood asthma (10). In other words, neonatal phototherapy may be postulated as a potential risk profile for asthma. However, the association between childhood asthma and neonatal phototherapy remains as a matter of debate.

\section{Objectives}

This study attempted to investigate the

\footnotetext{
Copyright $(\odot 2022$ The Author(s); Published by Nickan Research Institute. This is an open-access article distributed under the terms of the Creative Commons Attribution License (http://creativecommons.org/licenses/by/4.0), which permits unrestricted use, distribution, and reproduction in any medium, provided the original work is properly cited.
} 
relationship between childhood asthma and phototherapy.

\section{Patients and Methods}

\section{Study design}

This case-control study was performed on children aged between 2 and 14 years old with a definitive diagnosis of childhood asthma who were referred to Besat hospital in Hamadan, Iran, between 2017 and 2018. A gender and age-matched group of children with no evidence of asthma or other respiratory problems were selected as the control group. The two groups were matched for the mode of delivery, birth weight, and the method of feeding by breastfeeding or formula. The inclusion criteria for the case group were as follows; age ranged from 2 to 14 years old, gestational age higher than 36 weeks, the occurrence of jaundice with unknown origin (neonatal jaundice), no history of smoking, no history of familial asthma, and no history of blood transfusion due to asthma. The hospital's recorded files were the primary sources for the information on demographics, history of asthma in neonates or his/ her parents, history and time of phototherapy, as well as the data on neonatal hyperbilirubinemia. The diagnosis of childhood asthma was performed based on episodic respiratory symptoms (dyspnea, cough and wheezing), objective lung function testing by spirometry, and clinical response to a treatment trial with an inhaled short-acting beta $_{2}$ agonist reliever. The control group participants were randomly selected from the children with no evidence of respiratory problems who were referred to the hospital due to simple non-respiratory disorders or four laboratory checking-up. The study endpoint was assessing the link between the history of phototherapy and the risk for childhood asthma.

\section{Statistical analysis}

The results were presented as mean \pm standard deviation (SD) of quantitative variables and were summarized by absolute frequencies and percentages for categorical variables.

Normality of data analyzed using the KolmogorovSmirnov test. Categorical variables were compared using chi-square test or Fisher's exact test when more than 20\% of cells with expected count of less than 5 were observed. $P$ values below 0.05 were set as the significant level.

\section{Results}

In total, 69 children suffering from asthma and 71 nonasthmatic children as the controls were included in this study. The baseline characteristics in both groups are summarized in Table 1. The two groups were matched for mean birth weight $(P=0.374)$, mean gestational age $(P=$ $0.217)$, gender $(P=0.083)$, average age $(P=0.057)$, rating of the child's birth in the family $(P=0.849)$, the prevalence of pregnancy-related complications $(P=0.446)$, mode of delivery $(P=0.092)$, history of smoking in parents $(P$ $=0.186)$, and type of feeding in infancy $(P=0.264)$. We observed no between-group difference in the method of phototherapy (in hospital, at home using the fluorescent instrument, or at home with fluorescent lamps) ( $P=$ 0.804). Moreover, we found no differences in the degree of jaundice, age of jaundice began, or time for phototherapy (Table 2). Comparing the history of phototherapy between the asthmatic and non-asthmatic groups showed a higher rate of phototherapy in the former group $(37.7 \%$ versus $11.3 \% ; P<0.001)$.

Accordingly, it was shown that the previous history of phototherapy could increase the risk for asthma up to four times $(\mathrm{OR}=4.76,95 \% \mathrm{CI}: 1.97-11.51 ; P<0.001)$.

\section{Discussion}

The increased risk for childhood asthma in the children who suffer from hyperbilirubinemia and underwent phototherapy has attracted enormous interest. Some authors attributed it to the hyperbilirubinemia itself, some

Table 1. Baseline characteristics of asthmatic and non-asthmatic groups

\begin{tabular}{|c|c|c|c|}
\hline Item & Asthmatic group & Non-Asthmatic group & $P$ value \\
\hline Birth weight, gram & $3196.38 \pm 470.63$ & $3120.42 \pm 481.56$ & 0.374 \\
\hline Gestational age, week & $39.55 \pm 0.99$ & $39.77 \pm 0.70$ & 0.217 \\
\hline Male gender, $\%$ & $53(76.8)$ & $44(61.97)$ & 0.083 \\
\hline Mean age, year & $6.74 \pm 3.04$ & $6.15 \pm 3.09$ & 0.057 \\
\hline Rate at birth & & & 0.849 \\
\hline First & $34(49.3)$ & $32(45.1)$ & \\
\hline Second & $27(39.1)$ & $26(36.6)$ & \\
\hline Higher & $8(11.6)$ & $13(18.3)$ & \\
\hline Complication during pregnancy, \% & $6(8.7)$ & $9(12.7)$ & 0.446 \\
\hline Cesarean delivery, $\%$ & $34(49.3)$ & $25(35.2)$ & 0.092 \\
\hline History of smoking, \% & $19(27.5)$ & $27(38.0)$ & 0.186 \\
\hline Type of feeding & & & 0.264 \\
\hline Breastfeeding & $57(82.6)$ & $60(84.5)$ & \\
\hline Formula & $5(7.2)$ & $3(4.2)$ & \\
\hline Mixed & $7(10.1)$ & $8(11.3)$ & \\
\hline
\end{tabular}


Table 2. The characteristics of phototherapy in both asthmatic and non-asthmatic groups

\begin{tabular}{lcc}
\hline Item & Asthmatic group & Non-Asthmatic group \\
\hline The place of phototherapy & & $\boldsymbol{P}$ value \\
\hline Hospital & $12(46.2)$ & 0.804 \\
\hline Home by fluorescent instrument & $6(23.0)$ & $1(12.5)$ \\
\hline Home by fluorescent lamps & $8(30.8)$ & $3(37.5)$ \\
\hline The mean degree of jaundice & $3.29 \pm 14.94$ & $3.00 \pm 14.50$ \\
\hline Age at jaundice onset, day & $4.65 \pm 2.69$ & $3.62 \pm 1.77$ \\
\hline Time for phototherapy, day & $4.46 \pm 3.02$ & $3.25 \pm 1.75$ \\
\hline
\end{tabular}

to other comorbidities accompanying asthma such as postnatal complications or other neonatal comorbidities, and some others directly to phototherapy. Based on our initial suggestion, the increased likelihood of asthma may be directly related to the history of phototherapy. Therefore, we could show the increased risk for asthma in those neonates with the history of phototherapy. In this regard, the history of phototherapy could increase the risk for asthma up to more than four times. Because all baseline variables were comparable in both groups, approving this suggestion required no multivariable regression modelling for adjusting baseline factors. As a conclusion, appearing asthma or its exacerbation is expected in those neonates who are suffering from hyperbilirubinemia and planning for phototherapy.

The causal association between phototherapy and the increased risk for asthma has been pointed in almost all similar studies. As indicated by Kuzniewicz et al in 2018, using phototherapy to prevent infants from reaching these modest TSB levels is unlikely to be protective against asthma. Of course, in their study, the critical role of hyperbilirubinemia in triggering asthma was not refused, but it was not also approved. In this regard, those children with a total serum bilirubin level of $\geq 18 \mathrm{mg} / \mathrm{dL}$ were not at an increased risk for asthma (11). Aspberg et al proved that infant phototherapy and, or icterus are the risk factors for children with asthma before the age of 12 years old.

Additionally, after controlling contraries, they showed the odds ratio of 1.30 for phototherapy and, or icterus. However, in our study, we obtained higher odds ratios indicating a higher risk for asthma following phototherapy (12). In a large study conducted in the United States by Huang et al, the asthma risks increased along with the increase of total maximum bilirubin levels of serum after 48 hours from childbirth. According to their observations, neonatal maximum total levels of serum bilirubin greater than $15 \mathrm{mg} / \mathrm{dL}$ will increase the risk of asthma up to $61 \%$ in children. However, because phototherapy was unavailable in their selected study population, the role of phototherapy was not assessed. Still, it seems that the risk for asthma might be directly associated with hyperbilirubinemia rather than phototherapy (13). Several studies showed that there is a relationship between phototherapy and/or neonatal jaundice and asthma. The range of odds ratios was from 1.30 to 1.64 after adjustment for confounders $(14,15)$. However, we achieved a significantly higher risk ratio mostly emphasizing the dominant role of phototherapy in triggering childhood asthma in our children population.

Generally, phototherapy is benign, and many longitudinal studies showed that it has no potential side effects; however, the mechanism related to its triggering effects on childhood asthma remains uncertain. In a systematic review by Castro-Rodriguez et al, wide spectrum risk factors have been identified as the main determinants for childhood asthma. In this regard, the role of maternal weight gain or obesity, maternal use of antibiotics or paracetamol, maternal stress, caesarean delivery, severe respiratory infection, indoor exposure to mold or fungi, and outdoor air pollution were more highlighted (16). Thus, whether phototherapy or hyperbilirubinemia predispose children to asthma or not, remains questionable. As shown in our study, the degree and age at occurring jaundice did not determine the risk for asthma; however, we recommend performing larger studies for weighting both variables as the main indicators for childhood asthma.

\section{Conclusion}

The history of phototherapy can increase the risk of childhood asthma. This causal effect is independent of the degree and age of occurring hyperbilirubinemia, as the main indications for phototherapy.

\section{Limitations of study}

The study was single-center and conducted on a relatively low sample size.

\section{Acknowledgement}

The authors of the paper express their sincere gratitude to deputy research of Hamadan University of Medical sciences and all patients who participated in this study.

\section{Authors' contribution}

MHE and FE were the principal investigator of the study. MKS, $\mathrm{MH}$ and $\mathrm{FE}$ were included in preparing the concept and design. RR revised the manuscript and critically evaluated the intellectual contents. All authors participated in preparing the final draft of the manuscript, revised the manuscript and critically evaluated the intellectual contents. All authors have read and approved the content of the manuscript and confirmed the accuracy or integrity 
of all part of the work.

Conflicts of interest

The authors declare no conflicts of interest

\section{Ethical issues}

The research followed the tenets of the Declaration of Helsinki. The Ethics Committee of Hamadan University of Medical Sciences approved this study. The institutional ethical committee at Hamadan University of Medical Sciences approved all study protocols (IR. UMSHA.REC.1397.364). Accordingly, written informed consent was taken from all participants or their parents before any intervention. This study was extracted from M.D thesis of Mehran Hajiloo at this university (Thesis \#9706273821). Moreover, ethical issues (including plagiarism, data fabrication, double publication) have been completely observed by the authors

\section{Funding/Support}

This study was the result of a thesis in Hamadan University of Medical Science (\#9706203586).

\section{References}

1. NCD Countdown 2030 collaborators. NCD Countdown 2030: worldwide trends in non-communicable disease mortality and progress towards Sustainable Development Goal target 3.4. Lancet. 2018;392:1072-88. doi: 10.1016/S01406736(18)31992-5.

2. Fischer GB, Sarria EE, Camargos $P$, et al. Childhood asthma in low and middle-income countries: Where are we now? Paediatr Respir Rev. 2019;31:52-57. doi: 10.1016/j.prrv.2018.10.002.

3. Mendenhall E, Kohrt BA, Norris SA, Ndetei D, Prabhakaran D. Non-communicable disease syndemics: poverty, depression, and diabetes among low-income populations. Lancet. 2017;389:951-963. doi: 10.1016/S0140-6736(17)30402-6.

4. Hoch HE, Houin PR, Stillwell PC. Asthma in Children: A Brief Review for Primary Care Providers. Pediatr Ann. 2019;48(3):e103-e109. doi:10.3928/19382359-20190219-01

5. Ferrante G, La Grutta S. The Burden of Pediatric Asthma. Front. Pediatr. 2018;6:186. doi:10.3389/fped.2018.00186

6. Dijk FN, Folkersma C, Gruzieva O, et al. Genetic risk scores do not improve asthma prediction in childhood. J
Allergy Clin Immunol. 2019;144:857-860.e7. doi:10.1016/j. jaci.2019.05.017

7. Wei CC, Lin $\mathrm{CL}$, Shen TC, Kao CH. Neonatal jaundice and risks of childhood allergic diseases: a population-based cohort study. Pediatr Res. 2015;78:223-30. doi: 10.1038/pr.2015.89.

8. Tham EH, Loo EXL, Goh A, Teoh OH, Yap F, Tan KH, Godfrey KM, Van Bever H, Lee BW, Chong YS, Shek LP. Phototherapy for neonatal hyperbilirubinemia and childhood eczema, rhinitis and wheeze. Pediatr Neonatol. 2019;60(1):28-34. doi: 10.1016/j.pedneo.2018.03.004

9. Eyada IK, El Saie AL, Ibrahem GA, Riad NM. Effect of phototherapy on B and $\mathrm{T}$ lymphocytes in Egyptian infants suffering from neonatal jaundice. Allergol Immunopathol (Madr). 2017;45:290-296. doi: 10.1016/j.aller.2016.09.003.

10. Lloyd CM, Hessel EM. Functions of T cells in asthma: more than just $\mathrm{T}(\mathrm{H}) 2$ cells. Nat Rev Immunol. 2010;10(12):838-848. doi: 10.1038/nri2870

11. Kuzniewicz MW, Niki H, Walsh EM, McCulloch CE, Newman TB. Hyperbilirubinemia, Phototherapy, and Childhood Asthma. Pediatrics. 2018;142(4):e20180662. doi: 10.1542/ peds.2018-0662

12. Aspberg S, Dahlquist G, Kahan T, Källén B. Confirmed association between neonatal phototherapy or neonatal icterus and risk of childhood asthma. Pediatr Allergy Immunol. 2010;21:733-9. doi: 10.1111/j.1399-3038.2010.01038.x.

13. Huang L, Bao Y, Xu Z, Lei X, Chen Y, Zhang Y, Zhang J. Neonatal bilirubin levels and childhood asthma in the US Collaborative Perinatal Project, 1959-1965. Am J Epidemiol. 2013;178:1691-7. doi: 10.1093/aje/kwt248.

14. Aspberg S, Dahlquist G, Kahan T, Källén B. Confirmed association between neonatal phototherapy or neonatal icterus and risk of childhood asthma. Pediatr Allergy Immunol. 2010;21:e733-9. doi: 10.1111/j.1399-3038.2010.01038.x.

15. Ku MS, Sun HL, Sheu JN, Lee HS, Yang SF, Lue KH. Neonatal jaundice is a risk factor for childhood asthma: a retrospective cohort study. Pediatr Allergy Immunol. 2012;23:623-8. doi: 10.1111/j.1399-3038.2012.01345.x.

16. Castro-Rodriguez JA, Forno E, Rodriguez-Martinez CE, Celedón JC. Risk and protective factors for childhood asthma: what is the evidence?. J Allergy Clin Immunol Pract. 2016;4:1111-2. doi: 10.1016/j.jaip.2016.05.003. 